\title{
Issues and Countermeasures of Microblog Sports Event Communication
}

\author{
Liguo Zheng \\ Taishan Medical University, Tai'an, 271000, China
}

\begin{abstract}
Keywords: Microblog, Sports event communication, Issue, Countermeasure
\end{abstract}
\begin{abstract}
Microblog is a network communication tool generated under the historical background of internet prevailing. New pattern of sports event communication was also generated with this new tool: microblog. While providing a new platform for sports event, microblog also integrates modern elements to communication of sports event. In the communication process, microblog communication is promoted due to integration of positive energy. At the same time, it also improves the attention of more netizens to sports event. On this basis, the author mainly discussed relevant issues of microblog in sports event communication and relevant countermeasures.
\end{abstract}

\section{Introduction}

With popularization of network, information obtainment with network has already become the most principal channel for people's information obtainment. In the process of information communication, microblog includes a quite wide face of communication, including sports event report. From the perspective of sports event report, microblog has already become a main report mode. Journalists utilize relevant features of microblog to convey the latest information to wide network users through microblog. Moreover, promptness and effectiveness of microblog information communication also effectively increase young people's attention to sports event via microblog. To give better play to the function of microblog in sports event communication, it is of great significance for discussion on the issues existing in microblog sports event communication and relevant countermeasures.

\section{Summary of Microblog and Sports Event Microblog}

\section{Microblog}

Microblog is also termed as micro blog. It is a platform used for information sharing, communication and obtainment based on user relationship. Users can build personal community through client-side, express their inner thoughts with some simple words, and realize instant communication and sharing. This pattern allows any person to read or forward. Some micro blogs can make the content richer by releasing multimedia, such as video or picture. In the earliest, microblog was popular in America. Twitter is the earliest microblog website created by America in 2006. In July, 2006, Twitter was open to the public. The word of Twitter has already become the pronoun of microblog. Netease microblog, Sina microblog and Tencent microblog are prevailing microblog services in the mainland.

\section{Sports Event Microblog}

Under normal conditions, sports event is mainly of sports competition. It is not of frequent occurrence or mainly of one-time theme. Sports event refers to collective activities with certain time limit. Sports event can promote the development of local tourism, improve local popularity, and advocate city image. At the same time, sports even can also have direct effects on the organizer's society, economy and environment, make more and more people to understand a certain region, and make local folk culture, specialties and features to be known by numerous microblog fans. Sports event microblog refers to the microblog focused on the sponsor, organizer and organization personnel of sports event and service events. Its contents include ticket information, event broadcasting, athlete condition and interesting competition sidelights. Sometimes, some knowledge contests and prize-giving guess activities will be developed as well, so as to fully mobilize fans' initiative, and 
utilize advantages of microblog to attract more audiences. This category of microblog can attract numerous soccer fans, and guide then for match watching in a better way. In the contemporary era, many Chinese web portals have already established sports microblog, such as Netease sports microblog, Sina sports microblog and Tencent microblog forum.

\section{Issues of Microblog in Sports Event Communication}

\section{Pattern of microblog is too traditional, and its content lacks innovation}

In the operation process, many sports event micro blogs await for sports event officials to realize information. Their contents lack innovation. Released information only includes pictures and words, failing to give full play to the role of microblog or further promote some effective sports information. Usually, some sports event micro blogs brush the screen through competition words, failing to reach their effect, or even resulting in adverse effect. Some fans unfollowed. For example, official microblog of "Chinese free combat vs professional Thai boxing" tweet only a piece of news every two days after launching the microblog. The total number of news is only around 600. Moreover, most contents were forwarded. Fewer contents are original.

\section{Network information contents are overflowed, and its truth cannot be guaranteed}

In the process of microblog communication, it is unnecessary for released information to be reviewed, so any person can realize his or her trivial matters. Moreover, microblog is featured by openness, anonymity and briefness, making microblog usually communicate some untrue information. As a result, it is impossible to realize accurate and effective information communication. At the same time, it is also unable to meet people's tracing for background information. The value of such microblog cannot be reflected. Eventually, users' information obtainment costs are increased, and information truth cannot be effectively guaranteed. At last, it may lead the public life to be caught in the situation of information overflow. Due to rise of microblog, many network virus spreaders realize some web links with Trojan horse virus on microblog. Once users click these links, their mobile phones will be infected with Trojan horse virus. Moreover, some swindlers realize some false winning information or websites through microblog, resulting in some property losses to netizens.

Sports event microblog has much junk information, deviating from service competition

The main purpose of sports event microblog is to communicate sports events, serve sports audiences, and service sports media. Sports microblog operators should make it definite for this purpose. The number of media microblog is not limited for microblog operators, so some sports event micro blogs copy or forward other ones irrelevant to sports event, such as famous quotation, chicken soup for the soul and star's news. These blogs only blindly pursue for topics and public opinions, only for the purpose of attracting more fans and further expanding the influence of these blogs. Actually, this only involves simply copying and pasting of words. As a result, some junk information is overflowed. Thus, it directly affects communication of sports event.

It is difficult for information search, and operation service is controversy

From foreign Twitter to domestic microblog websites, the operation pattern is complete for many microblog websites. User cultivation requires much costs and energy. In the process of user experience, it is necessary to obtain more high-quality users. However, it is only in the exploratory pattern of profit. At the same time, some clear-cut plans are not given. It is definite that users' difficulty in news searching will be improved to some extent by releasing some mass information every day. Thus, much time and energy of netizens is wasted. Compared with traditional media, communicating effect of microblog may not be as good as traditional media.

\section{Effective Countermeasures for Application of Microblog in Sports Event Communication}

Increase the contents in microblog information communication

The contents of microblog should be amiable, active and readable. Moreover, some pictures can be properly used, so as to enhance richness of microblog contents, and strengthen fans' cognition for microblog contents. Each microblog can be classified according to the title. Thus, it is convenient for 
fans to read and distinguish. Moreover, the topic pattern "\#” can be uploaded on the title, so as to eventually classify as a topic microblog. Thus, it is more convenient for communication in microblog. At the same time, fans can also search according to their hobbies. For operation and management of sports microblog, emphasis should be laid on mastering of details. For instance, quotation of the contents from other's microblog must be marked. Thus, respect to the originator can be reflected. Moreover, interaction with others on microblog can be enhanced as ell. It is necessary for sports event microblog to attach importance to richness and diversity of its contents, proportion between forwarded and original news, and improvement to the number of original news. Thus, it can effectively prevent reprinting and copy. According to Li Kaifu, the contents of microblog should be original and forwarded. To operate sports microblog, we should properly forward news in other aspects. Thus, internet groups with attention to sports event can be continuously expanded. Moreover, interestingness of network reading can be intensified by forwarding some other news, especially some interesting things about sports event, making it more popular for the microblog communication pattern of sports event.

\section{Strengthen website construction and guarantee content effectiveness}

Each microblog website must manage its microblog information. Network management persons need to face mass microblog information every day, including true \& false information, speculation news and sensational news. Thus, microblog management persons are required to filter and screen such information, and select effective microblog information and topics that wide fans are interested in. microblog website needs to actively and positively establish a systematic management mode for the convenience of further improvement to system management capacity. Besides, we also need to reserve a small block on microblog website to realize some meaningful information. Microblog users can obtain practical news from this block. At the same time, microblog website can also set up a reward mechanism. For bloggers with much and wonderful contents, some substantial rewards can be given, so as to improve their pursuit for microblog quality and content.

\section{Enhance interaction with microblog netizens, and intensify relevant services}

In the use process of microblog, a very important feature and link is feedback and interaction between micro blogs. If without feedback and interaction, the information communicator doesn't know whether the receiver received the information or not and whether the receiver understood his intention. Feedback is a process responsible for communication adjustment as well as a process for further strengthening communication. To obtain better development and obtain fans' recognition, it is far from enough only by presenting its contents to fans. It is also necessary to show the posture of listening, lay more importance on audiences' information feedback, and positively convey feedback information to sports event organizers. Thus, relevant works of sports event can be promoted. Moreover, it is also necessary to emphasize effective interaction with fans with "weak ties", so as to bring better benefits to sports event and obtain unexpected effect. The reason is that most fans with "weak ties" have abundant information and interpersonal connections. At the same time, their forward and comments of microblog information will present unexpected communication speed of sports event. Therefore, it is essential for development of fans with "weak ties". Effective interaction exists between sports event microblog and fans. We can use for reference experience of business microblog, and encourage fans to forward and make comments through a series of award forwarding activities. Thus, influence and communication of microblog can be expanded. Moreover, the sponsor's brand can be promoted in many aspects, so as to improve the sponsor's brand awareness.

\section{Broaden profit channels of microblog website}

According to some people, microblog website takes profit as the main purpose. At first, microblog website fights for users, and then makes profit. Considering not many available advertisement resources, it is not very high for limitation to user's reception features and user terminals used to distinguish advertisement contents. However, most microblog websites don't directly adopt the mode of profit by advertisement. As estimated, such websites will not profit from advertisement contents for a long period of time. However, it is different for web portals. It is a good business opportunity to hold some large-scale sports events. Microblog can be completely applied into sports event, so as to further expand the profit system of web portals. Web portals can properly use search performance, 
provide some real-time microblog search services in various aspects, and convert some messy information into valuable information. As long as microblog has development potential and users have certain needs, such potential value will be given full play to someday, obtaining more profits for microblog operators.

\section{Conclusion}

To sum up, communication of sports event in microblog is the same as other information communications. As long as it has features of microblog, it is unnecessary for professional training on information communication or more media conversion rights. Thus, it reduces the threshold for sports event report in microblog, beneficial for communication of sports event. At the same time, it is also beneficial for more sports fans to know the latest information through microblog. Fast and convenient information communication of microblog allows sports microblog to receive wide attention from wide microblog fans since its appearance. However, low threshold of microblog information communication also brings forth many adverse impacts on spots event communication, such as insufficient supervision of information communication, information overflow and insufficient depth of information communication. This also causes bad impacts on communication of sports event. Therefore, it is imperative for strengthening network supervision. While finding any false information, we should promptly filter, so as to guarantee authenticity of sports event communication.

\section{References}

[1] Yin Guohua, Sina Microblog: Innovation to New Pattern of Sports Event Report, Ad Men, 2010, (08): 94-95;

[2] Huang Haiyan and Zhang Lin, Research on Basic Theory of Sports Event - Discussion on of Historical Development, Definition, Classification and Characteristics of Sports Event, Journal of Wuhan Institute of Physical Education, 2011, (02): 24-25;

[3] Zhou Bei, Discussion on Application and Development of New Media (Microblog) in Sports News Report of London Olympic Games, Science \& Technology of Stationery \& Sporting Goods, 2013, (03): 42-43;

[4] Chen Changfeng and Qiu Junxi, Microblog Communication: Strength of "Weak Ties” and Collective Intelligence, Journalism Lover, 2013, (03): 18-19;

[5] Mingjun Xin,Hanxiang Wu,Zhihua Niu. A Quick Emergency Response Model for Micro-blog Public Opinion Crisis Based on Text Sentiment Intensity[J]. Journal of Software. 2012 (6)

[6] Tao Ran and Zhao Yun, Research on Sports Event Report in Media Era - With Southern Urban Daily as an Example, Journal of Sports Adult Education, 2012, (03): 112-114. 